\title{
A 36-week multicenter, randomized, double-blind, placebo-controlled, parallel- group, phase 3 clinical trial of sodium oligomannate for mild-to-moderate Alzheimer's dementia
}

Shifu Xiao ${ }^{1,2^{*}+}$ (D) Piu Chan ${ }^{3+}$, Tao Wang ${ }^{1,2+}$, Zhen Hong ${ }^{4}$, Shuzhen Wang ${ }^{5}$, Weihong Kuang ${ }^{6}$, Jincai He ${ }^{7}$, Xiaoping Pan ${ }^{8}$, Yuying Zhou ${ }^{9}$, Yong $\mathrm{Ji}^{9}$, Luning Wang ${ }^{10}$, Yan Cheng ${ }^{11}$, Ying Peng ${ }^{12}$, Qinyong Ye ${ }^{13}$, Xiaoping Wang ${ }^{14}$, Yuncheng $\mathrm{Wu}^{14}$, Qiumin $\mathrm{Qu}^{15}$, Shengdi Chen ${ }^{16}$, Shuhua $\mathrm{Li}^{17}$, Wei Chen ${ }^{18}$, Jun $\mathrm{Xu}^{19}$, Dantao Peng ${ }^{20}$, Zhongxin Zhao ${ }^{21}$, Yansheng Li ${ }^{22}$, Junjian Zhang ${ }^{23}$, Yifeng Du ${ }^{24}$, Weixian Chen ${ }^{25}$, Dongsheng Fan ${ }^{26}$, Yong Yan ${ }^{27}$, Xiaowei Liü ${ }^{28}$, Wei Zhang ${ }^{29}$, Benyan Luo ${ }^{30}$, Wenyuan $\mathrm{Wu}^{31}$, Lu Shen ${ }^{32}$, Chunfeng Liu ${ }^{33}$, Peixian Mao ${ }^{34}$, Qiumei Wang ${ }^{35}$, Qianhua Zhao ${ }^{4}$, Qihao Guo ${ }^{4}$, Yongtao Zhou ${ }^{3}$, Yi Li ${ }^{5}$, Lijun Jiang ${ }^{6}$, Wenwei Ren ${ }^{7}$, Yingjun Ouyang ${ }^{8}$, Yan Wang ${ }^{9}$, Shuai Liu', Jianjun Jia ${ }^{10}$, Nan Zhang ${ }^{11}$, Zhonglin Liu' ${ }^{12}$, Raoli He ${ }^{13}$, Tingyi Feng ${ }^{14}$, Wenhui Lu ${ }^{15}$, Huidong Tang ${ }^{16}$, Ping Gao ${ }^{17}$, Yingchun Zhang ${ }^{18}$, Lanlan Chen ${ }^{19}$, Lei Wang ${ }^{20}$, You Yin ${ }^{21}$, Qun Xu ${ }^{22}$, Jinsong Xiao ${ }^{23}$, Lin Cong ${ }^{24}$, Xi Cheng ${ }^{25}$, Hui Zhang ${ }^{26}$, Dan Gao ${ }^{27}$, Minghua Xia ${ }^{28}$, Tenghong Lian ${ }^{29}$, Guoping Peng ${ }^{30}$, Xu Zhang ${ }^{31}$, Bin Jiao ${ }^{32}$, Hua Hu ${ }^{33}$, Xueyan Chen ${ }^{34}$, Yihui Guan ${ }^{4}$, Ruixue Cui ${ }^{35}$, Qiu Huang ${ }^{36}$, Xianliang Xin ${ }^{37}$, Hongjian Chen ${ }^{37}$, Yu Ding ${ }^{37}$, Jing Zhang ${ }^{37}$, Teng Feng ${ }^{37}$, Marc Cantillon ${ }^{37}$, Kewei Chen ${ }^{38}$, Jeffrey L. Cummings ${ }^{39}$, Jian Ding ${ }^{40}$, Meiyu Geng ${ }^{40^{*}}$ and Zhenxin Zhang ${ }^{35^{*}}$

\section{Abstract}

Background: New therapies are urgently needed for Alzheimer's disease (AD). Sodium oligomannate (GV-971) is a marine-derived oligosaccharide with a novel proposed mechanism of action. The first phase 3 clinical trial of GV971 has been completed in China.

(Continued on next page)

\footnotetext{
*Correspondence: xiaoshifu@msn.com; mygeng@simm.ac.cn; wuzhangzhenxin@163.com

'Shifu Xiao, Piu Chan and Tao Wang contributed equally to this work. 'Department of Geriatric Psychiatry, Shanghai Mental Health Center, Shanghai Jiaotong University School of Medicine, Shanghai, China

${ }^{40}$ State Key Laboratory of Drug Research, Shanghai Institute of Materia

Medica, Chinese Academy of Sciences, 555 Zu chong zhi Road, Nevada,

China

${ }^{35}$ Peking Union Medical College Hospital, No. 1 Shuaifuyuan, Beijing 100730,

China

Full list of author information is available at the end of the article
}

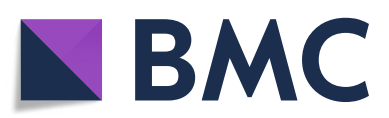

(c) The Author(s). 2021 Open Access This article is licensed under a Creative Commons Attribution 4.0 International License, which permits use, sharing, adaptation, distribution and reproduction in any medium or format, as long as you give appropriate credit to the original author(s) and the source, provide a link to the Creative Commons licence, and indicate if changes were made. The images or other third party material in this article are included in the article's Creative Commons licence, unless indicated otherwise in a credit line to the material. If material is not included in the article's Creative Commons licence and your intended use is not permitted by statutory regulation or exceeds the permitted use, you will need to obtain permission directly from the copyright holder. To view a copy of this licence, visit http://creativecommons.org/licenses/by/4.0/ The Creative Commons Public Domain Dedication waiver (http://creativecommons.org/publicdomain/zero/1.0/) applies to the data made available in this article, unless otherwise stated in a credit line to the data. 
(Continued from previous page)

Methods: We conducted a phase 3, double-blind, placebo-controlled trial in participants with mild-to-moderate AD to assess GV-971 efficacy and safety. Participants were randomized to placebo or GV-971 (900 mg) for 36 weeks. The primary outcome was the drug-placebo difference in change from baseline on the 12-item cognitive subscale of the Alzheimer's Disease Assessment Scale (ADAS-cog12). Secondary endpoints were drug-placebo differences on the Clinician's Interview-Based Impression of Change with caregiver input (CIBIC+), Alzheimer's Disease Cooperative Study-Activities of Daily Living (ADCS-ADL) scale, and Neuropsychiatric Inventory (NPI). Safety and tolerability were monitored.

Results: A total of 818 participants were randomized: 408 to GV-971 and 410 to placebo. A significant drug-placebo difference on the ADAS-Cog12 favoring GV-971 was present at each measurement time point, measurable at the week 4 visit and continuing throughout the trial. The difference between the groups in change from baseline was -2.15 points (95\% confidence interval, -3.07 to -1.23 ; $p<0.0001$; effect size 0.531 ) after 36 weeks of treatment. Treatment-emergent adverse event incidence was comparable between active treatment and placebo (73.9\%, 75.4\%). Two deaths determined to be unrelated to drug effects occurred in the GV-971 group.

Conclusions: GV-971 demonstrated significant efficacy in improving cognition with sustained improvement across all observation periods of a 36-week trial. GV-971 was safe and well-tolerated.

Trial registration: ClinicalTrials.gov, NCT02293915. Registered on November 19, 2014

Keywords: Sodium oligomannate, Efficacy, Safety, Alzheimer's disease, Clinical trial

\section{Background}

Alzheimer's disease (AD) is the most common type of dementia in older people. According to the World Alzheimer Report 2018, approximately 50 million people worldwide are currently suffering from dementia, and two thirds of them have AD. China has more than 6 million $\mathrm{AD}$ patients, and this number is expected to exceed 10 million by 2050 [1, 2]. AD has become a major cause of disability and death among older individuals. AD has a devastating social and economic impact on patients, their families, their caregivers, medical systems, and society. Several hundred treatments for AD have been tested in clinical trials, but only four [3-6] have been authorized and widely used for $\mathrm{AD}$ treatment. Although repeated failures of phase 3 clinical trials in the last decade have been reported $[7,8]$, much effort continues to be invested in developing treatments for this disease [9].

Sodium oligomannate (GV-971) is a marine-derived oligosaccharide and a mixture of linear, acidic oligosaccharides with a degree of polymerization ranging from dimers to decamers [10]. After oral administration, most of the ingested GV-971 is retained in the gut. It is proposed that it can reconstitute the gut microbiota, reduce bacterial metabolite-driven peripheral infiltration of immune cells into the brain, and inhibit neuroinflammation in the brain as observed in animal models [11]. Some GV-971 can penetrate into the brain and directly inhibit $\mathrm{A} \beta$ fibril formation and destabilizes the preformed fibrils into non-toxic monomers. It reduces $A \beta$ deposition in the brain of $A \beta$-transgenic mice [12-15]. The reduction in both $A \beta$ deposition and neuroinflammation may synergistically contribute to the improvement of cognitive function observed in multiple non-clinical models. Although the above-proposed mechanism of action requires validation in humans, the non-clinical mechanistic studies have demonstrated that the properties of GV-971 make it a candidate anti-AD therapy. Phase 1 and phase 2 studies demonstrated that GV-971 is safe and well-tolerated, and $450 \mathrm{mg}$ twice-a-day (b.i.d.) was selected as phase 3 dosage [16].

Based on the results and experience from the phase 2 trial, we designed and completed the phase 3 trial. The phase 3 trial reported here was undertaken to further evaluate the efficacy and safety of GV-971 in participants with mild-to-moderate AD.

\section{Methods \\ Study design}

This phase 3 study was a 36-week multicenter, randomized, double-blind, placebo-controlled parallel-group clinical trial. Participants with mild-to-moderate $A D$ were assigned randomly (1:1 ratio), to receive GV-971 (450 mg, b.i.d.) or placebo.

The trial protocol was approved by the Ethics Review Board of Shanghai Mental Health Center (Shanghai, China) and is registered on https://clinicaltrials.gov/ NCT02293915. The protocol was also approved by the Institutional Review Boards of all participating sites. The Clinical New Drug Research and Development Team of the Department of Psychogeriatrics of Shanghai Mental Health Center and the sponsor designed the study in consultation with academic advisors. All participants or their representatives provided written informed consent before participation in the trial. 
Data were gathered by the study investigators through the Merge system (www.merge.com/eClinical), analyzed by IQVIA (Durham, NC, USA) after the data were locked, and interpreted by the academic main investigators in collaboration with the sponsor. The academic authors attest to the accuracy and integrity of the data and the fidelity of this report to the study protocols, which are available with the full protocol (protocol number: 971-III; version 7.1) and statistical analysis plan (supplementary appendix).

There were 8 protocol amendments and 4 protocol versions in the course of the trial. A planned 24-week interim analysis was deleted, and the study continued to its primary outcome at 36 weeks. The other amendments included deletion of the age adjustments on the interpretation of the Fazekas scale of allowable white matter pathology on magnetic resonance imaging (MRI) and adjustment in how routine laboratory studies were interpreted. The subject whose Mini-Mental State Examination (MMSE) [17] fluctuation was more than 2 score from screening visit to randomize visit should be double-checked according to the inclusion/exclusion criteria in order to accurately enroll the subjects participant. Allowable heart rates were broadened (to $>55$ beats per minute), and apolipoprotein E (APOE) genotyping was made mandatory. The intended sample size of 788 was somewhat over-recruited resulting in a final sample size of 818 randomized subjects.

\section{Participants}

Participants were aged $50-85$ years of age and met the diagnostic criteria for probable AD according to the $\mathrm{Na}$ tional Institute of Neurological and Communicative Disorders and Stroke and the Alzheimer's Disease and Related Disorders Association [18]. Participants had mild-to-moderate $\mathrm{AD}$, with a MMSE score from 11 to 22 inclusive for participants with only a primary school education and from 11 to 26 inclusive for those with an education beyond primary school, in line with representative China national sample of age, gender, education reference norms $[17,19]$. A total Hachinski Ischemia Scale [20] score of $\leq 4$ was required, and the Hamilton Hamilton Depression Scale 17 [21] score had to be $\leq 10$.

We strengthened brain MRI assessment in the phase 3 trial. During the screening, brain magnetic resonance imaging (MRI) with oblique coronal hippocampus images was undertaken; a medial temporal lobe atrophy visual rating scale score $\geq 2$ was required [22]. MRI data were sent to an imaging advisory team via email in digital imaging and communications in medicine format along with a brief medical history. A Fazekas scale [23] for white matter lesions of grade $\geq 3$ (moderate-to-severe) or $>2$ lacunar infarction lesions of diameter 1.0$2.0 \mathrm{~cm}$ or infarction lesions in vital brain areas (thalamus, hippocampus, or entorhinal cortex) excluded participation. The brain MRI results of all participants were presented for diagnostic review by the imaging advisory team to minimize diagnostic error. The investigators reviewed the laboratory examination results and comments from the imaging advisory team on the brain MRI examination to ascertain if participants met the enrollment criteria.

Female participants were postmenopausal (last menopause at least 24 weeks prior to study entry), surgically sterilized, or of childbearing age who agreed to use contraceptive measures during the trial. Women of childbearing age and women $<24$ weeks from the start of menopause underwent a urine pregnancy test during screening, and the result was required to be negative for study entry.

Participants were required to have completed education of at least primary school level or higher and be able to complete the protocol-specified cognitive tests. Progressive impairment of memory must have been present for $\geq 12$ months. Care partners for the participants had frequent contact ( $\geq 4$ days every week for $\geq 2 \mathrm{~h}$ on each of these days). Caregivers had to be willing to provide critical trial data on caregiver-based scales: CIBIC+ [24, 25], Alzheimer's Disease Cooperative StudyActivities of Daily Living (ADCS-ADL) scale [26], and Neuropsychiatric Inventory (NPI) [27, 28]. Before implementation of a protocol-related procedure or examination, participants and caregivers provided written informed consent. If participants could not sign due to limited cognition, their legal guardians signed on their behalf.

Participants were excluded if they had taken part in another clinical trial $<30$ days before the initiation of this trial or had dementia due to non-AD causes. Participants had a normal neurologic examination. They were excluded if they had abnormal laboratory values. They were also excluded if they had unstable or severe cardiac, pulmonary, hepatic, renal, or hematopoietic disease (including unstable angina, uncontrolled asthma, active gastric bleeding, or cancer); a resting heart rate $<55 \mathrm{bpm}$ after $10 \mathrm{~min}$ of rest; a visual/hearing disorder that prevented completion of neuropsychologic tests and scale evaluations; or a history of alcohol abuse, drug abuse, or severe psychopathology (including major depression). Participants could not be on cholinesterase inhibitors or memantine while enrolled in this trial and were required to be off these agents for $\geq 4$ weeks before randomization. The investigators excluded participants who they thought could not complete this trial, who participated in the phase 2 GV-971 trial, who were on $\mathrm{AD}$ therapies that could not be stopped, or were relatives of staff of IQVIA or Shanghai Green Valley Pharmaceuticals. 
This clinical trial was conducted at 34 participating sites in the psychiatry, neurology, and geriatric departments of hospitals in several regions of China. $\left[{ }^{18} \mathrm{~F}\right]-$ FDG-PET was carried out at two sites (Beijing and Shanghai) where appropriate technology and expertise were routinely available.

\section{Interventions}

One capsule provides $150 \mathrm{mg}$ of GV-971. The placebo is identical to the GV-971 capsule with regard to taste, odor, appearance, and design and is acceptable based on the quality standard inspection approved by the National Medical Products Administration (NMPA). GV-971 capsules are manufactured according to the Good Manufacturing Practice (GMP).

Two groups were intervened by GV-971 and placebo. The study comprised a 2-week screening period, 4-week run-in period, and 36-week treatment period. During the run-in period, each participant took three placebo capsules b.i.d. During the 36-week treatment period, each participant took three GV-971 capsules $(150 \mathrm{mg} /$ capsule) or three placebo capsules b.i.d.

Drug distribution was undertaken according to the drug randomization number generated by an interactive web response system (IWRS). After participants were determined to be eligible for trial participation, investigators logged into the IWRS system to obtain a randomization number and drug kit number for each participant. During the 36-week treatment period, participants were randomized (1:1 ratio) to receive GV-971 ( $450 \mathrm{mg}$, b.i.d.) or the matching placebo. During each on-site visit (Figure S3), investigators recorded the distribution and return of trial drugs. Participants, caregivers, and trial staff remained blinded to treatment assignment throughout the trial.

\section{Outcomes}

The primary efficacy endpoint was the drug-placebo difference in the change from baseline on the ADASCog12 (score range, 0-75, with higher scores indicating greater cognitive impairment) at week 36 [29]. Secondary endpoints were intergroup differences including CIBIC+ $[24,25]$ and changes from baseline in the ADCS-ADL scale (score range, $0-78$, with lower scores indicating worse functioning) [26] and NPI (score range, 0-144, with lower scores indicating fewer behavioral disturbances) [27, 28]. Intergroup differences in change from baseline in a global index defined for relative cerebral metabolic rate for glucose (CMRglu) on $\left[{ }^{18} \mathrm{~F}\right]-\mathrm{FDG}$ PET [30] after 36 weeks of double-blind treatment was measured in a subgroup of participants. There was no change in the choice of and prioritization of outcome measures in the course of the trial.
From the time the subject signs the informed consent form, all adverse events (AEs) that occurred were captured on case report forms and included in the summaries. AE evaluation comprised classification of the organ system, grade, relationship to drug exposure, action taken to the treatment (if any), and the outcome.

Ten on-site visits and four telephone interviews were conducted with each participant. The on-site visits occurred at weeks -6 and -4 , day 0 , and weeks $4,8,12$, $16,24,36$, and 40 . Telephone interviews were undertaken at weeks $2,20,28$, and 32 .

\section{Sample size}

The sample size was calculated with nQuery Advisor 7.0. According to the results of the GV-971 phase 2 trial and other anti-AD clinical trials, a minimum ADAS-Cog drug-placebo difference of 1.4 was assumed. Completion of the trial by 315 participants in each arm would provide $80 \%$ power to detect a standardized effect size (difference/SD) of $\geq 0.233$ between the GV-971 and placebo groups with a two-sided alpha level of 0.05 . Therefore, we planned to enroll 788 participants, allowing for an anticipated $20 \%$ dropout rate. In total, 818 participants were randomized.

\section{Data statistics and study parameters}

The primary endpoint (change from baseline of the ADAS-Cog12 score at week 36 in the treatment group compared to the placebo group) was analyzed using analysis of covariance (ANCOVA) with the treatment group, education level, pooled center, and age group as fixed effects and the baseline MMSE score and baseline ADAS-Cog12 score as covariates. Control-based pattern imputation was used to supply the missing data in the primary analysis. In general, this method assumed that, after trial withdrawal, participants from the treatment arm would exhibit the same future evolution of the disease as participants receiving the control treatment. Participants who discontinued the trial from the control arm were assumed to evolve in the same way as control participants who remained in the trial. In addition, a mixed-model repeated-measures model (MMRM) was used for sensitivity analyses. The primary efficacy analysis was based on the full analysis set (FAS), which included all randomly assigned participants who received at least one dose of the double-blind study drug and had a baseline value and at least one post-baseline efficacy assessment.

For secondary endpoints, CIBIC+ was analyzed by a stratified Cochran-Mantel-Haenszel test, including the stratification factors of MMSE at baseline, education level, and age group. ANCOVA was used to assess the drug-placebo difference in the change from baseline on ADCS-ADL and NPI scores. 
Safety analyses were based on the safety analysis set, which included all randomly assigned participants who received at least one dose of a study agent. Safety analyses were based on a summary of AEs, laboratory assessments, electrocardiograms, vital signs, and physical examinations.

Participants in the cerebral $\left[{ }^{18} \mathrm{~F}\right]$-FDG-PET sub-cohort underwent PET at baseline and week 36 . We used the statistical region of interest (sROI) approach to compute the relative global CMRglu global index [30]. sROI was introduced based on cross-validation and ADNI data and was used in a recent clinical trial [31]. The sROIdefined global CMRglu index is actually the standard uptake value ratio between a set of regions affected by $\mathrm{AD}$ and a set of regions spared by $\mathrm{AD}$ in terms of glucose uptake decline over time [30].

SAS v9.4 (SAS Institute, Cary, NC, USA) was used for statistical analyses. $p<0.05$ (two-sided) indicated significance.

\section{Results}

\section{Participant flow}

The screening, randomization, and follow-up of participants are summarized in Fig. 1. The trial was initiated in March 2014 and completed in June 2018. The trial was completed as planned after the last participant randomized had completed the exposure period and final visit measures. Among the 1291 participants screened, 818 participants were randomized, and 817 received at least one dose of the study treatment (408 were assigned randomly to the GV-971 group and 410 to the placebo group). Screen failures were 390 (30.2\%). A total of 901 participants were included in the run-in period. Run-in failures were 83 (6.4\%). The two main reasons for screen failure and run-in failure were that (1) MRI findings did not meet the inclusion criteria with 205 (43.3\% of screen failures) and (2) 70 (14.8\% of screen failures) participants/legal guardians withdrew consent (Fig. 1). The completion rate was $81.9 \%$ for participants in the GV971 group and $83.9 \%$ for the placebo group. The most common reasons for early termination were withdrawal of consent and AEs, regardless of group assignment (Fig. 1).

\section{Baseline data}

The demographic and baseline characteristics of the trial cohort are summarized in Table 1 . There were no significant differences between the GV-971 and placebo groups with respect to age, sex, ethnicity, or education level (Table 1). In total, $52.5 \%$ of participants in the GV971 group and $48.5 \%$ in the placebo group were apolipoprotein E epsilon 4 (APOE $\varepsilon 4$ ) carriers. The mean \pm SD MMSE score was $19.4 \pm 4.4$ in the GV-971 group and $19.5 \pm 4.5$ in the placebo group. The mean \pm SD duration (in months) from symptom onset was $30.42 \pm 20.59$ in the GV-971 group and $31.46 \pm 20.79$ in the placebo group. There were no significant differences between the groups in baseline characteristics including age, race, education, APOE4 distribution, ADAS-Cog12, ADCS$\mathrm{ADL}$, and NPI scores.

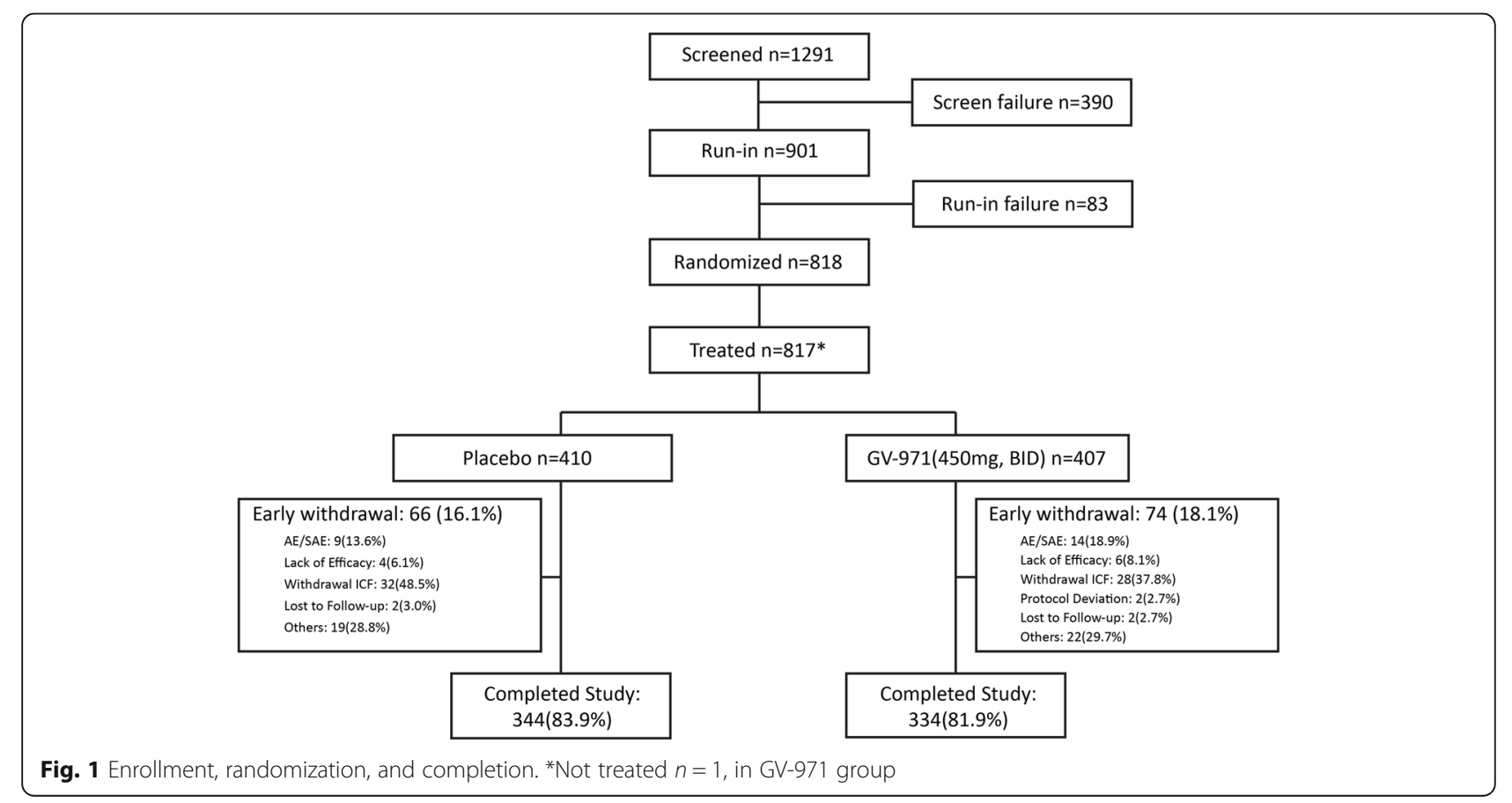


Table 1 Demographic and baseline clinical characteristics

\begin{tabular}{|c|c|c|c|}
\hline Characteristic & Placebo, $n=410$ (\%) & GV-971, $n=408$ (\%) & $p$ value \\
\hline Age-years & $69.7 \pm 8.20$ & $69.6 \pm 8.12$ & $0.7374[b]$ \\
\hline$\leq 65$ years & $131(32.0 \%)$ & $132(32.4 \%)$ & $0.9021[\mathrm{c}]$ \\
\hline$>65$ years & $279(68.0 \%)$ & $276(67.6 \%)$ & \\
\hline Sex & & & $0.8242[c]$ \\
\hline Male-no. (\%) & $177(43.2 \%)$ & $173(42.4 \%)$ & \\
\hline Female—no. (\%) & $233(56.8 \%)$ & $235(57.6 \%)$ & \\
\hline Han ethnicity—no. (\%) & $402(98.0 \%)$ & $398(97.5 \%)$ & $0.6261[\mathrm{c}]$ \\
\hline Education-years & & & $0.7394[\mathrm{c}]$ \\
\hline$>6$ years & $335(81.7 \%)$ & $337(82.6 \%)$ & \\
\hline$\leq 6$ years & 75 (18.3\%) & $71(17.4 \%)$ & \\
\hline APOE ع4 carrier-no. (\%) & $199(48.5 \%)$ & $214(52.5 \%)$ & $0.2758[\mathrm{c}]$ \\
\hline MMSE score & $19.5 \pm 4.5$ & $19.4 \pm 4.4$ & $0.5795[b]$ \\
\hline MMSE $<11$ & $1(0.2 \%)$ & 0 & $0.6815[\mathrm{~d}]$ \\
\hline $11 \leq \mathrm{MMSE}<15$ & $72(17.6 \%)$ & $68(16.7 \%)$ & \\
\hline $15 \leq$ MMSE $\leq 19$ & $118(28.8 \%)$ & $122(29.9 \%)$ & \\
\hline $20 \leq \mathrm{MMSE} \leq 26$ & $219(53.4 \%)$ & $216(52.9 \%)$ & \\
\hline $26<$ MMSE & 0 & $2(0.5 \%)$ & \\
\hline Duration since symptom onset (months) & $31.46 \pm 20.79$ & $30.42 \pm 20.59$ & $0.6087[b]$ \\
\hline ADAS-Cog12 & $20.88 \pm 10.00$ & $21.28 \pm 10.14$ & $0.5638[b]$ \\
\hline ADCS-ADL & $64.2 \pm 10.1$ & $64.0 \pm 11.2$ & $0.8910[b]$ \\
\hline NPI & $5.9 \pm 8.6$ & $5.6 \pm 8.0$ & $0.5651[\mathrm{~b}]$ \\
\hline
\end{tabular}

[a] refers to the $t$ test; [b] refers to the Wilcoxon rank sum test; [c] refers to the chi-square test; [d] refers to Fisher's exact test

\section{Outcomes}

The results of the primary and secondary analyses are summarized in Table 2 and Figs. 2, 3, and 4. Changes from baseline over time in ADAS-cog12 scores are shown in Fig. 2. The mean changes from baseline at week 36 were -2.70 points for the GV-971 group and 0.16 points for the placebo group, with an unadjusted group difference (GV-971 group minus placebo group) of -2.54 points. The mean modeled difference between the groups (GV-971 group minus placebo group) with regard to the change from baseline to week 36 was 2.15 points $(95 \%$ confidence interval, -3.07 to -1.23 ; $p<0.0001$, with Cohen's $d$ effect size 0.53 , using pooled SD of change score ANCOVA analysis. There were no significant drug-placebo differences for prespecified secondary outcomes. The $p$ value of CIBIC+ was 0.059

Table 2 Primary and secondary outcomes

\begin{tabular}{|c|c|c|c|c|c|c|c|c|}
\hline \multirow[t]{2}{*}{ Outcome } & \multicolumn{2}{|c|}{ Raw score at baseline } & \multicolumn{2}{|c|}{ Raw score at week 36} & \multicolumn{2}{|c|}{ Least square mean change at week $36(95 \% \mathrm{Cl})$} & \multirow{2}{*}{$\begin{array}{l}\text { Estimated } \\
\text { difference at } \\
\text { week } 36(95 \% \mathrm{Cl})\end{array}$} & \multirow[t]{2}{*}{$p$ value } \\
\hline & Placebo & GV-971 & Placebo & GV-971 & Placebo & GV-971 & & \\
\hline \multicolumn{9}{|l|}{ Primary } \\
\hline ADAS-Cog $12^{*}$ & $20.83 \pm 10.03$ & $21.30 \pm 10.12$ & $20.55 \pm 11.93$ & $18.32 \pm 10.71$ & $0.26(-0.58,1.10)$ & $-1.89(-2.78,-1.00)$ & $-2.15(3.07,-1.23)$ & $<0.0001$ \\
\hline \multicolumn{9}{|l|}{ Secondary } \\
\hline $\mathrm{CIBIC}+* *$ & - & - & $4.0 \pm 0.85$ & $3.9 \pm 0.83$ & & & & 0.0588 \\
\hline ADCS-ADL & $64.2 \pm 10.1$ & $64.0 \pm 11.2$ & $63.4 \pm 11.4$ & $63.5 \pm 11.6$ & $-1.41(-2.18,-0.64)$ & $-1.15(-1.95,-0.35)$ & $0.26(-0.64,1.16)$ & 0.57 \\
\hline NPI & $5.9 \pm 8.7$ & $5.6 \pm 8.0$ & $4.8 \pm 8.6$ & $5.0 \pm 7.7$ & $-0.11(-0.93,0.71)$ & $0.01(-0.84,0.87)$ & $0.12(-0.84,1.09)$ & 0.80 \\
\hline
\end{tabular}

The plus-minus values for the scores at baseline and at 36 weeks are means ( \pm SD). Least square means are estimated using ANCOVA, with the treatment group, education level, pooled center, and age group as fixed effects, and baseline MMSE level and baseline score as covariates. The estimated difference is the least square mean change from baseline between the two groups (GV-971 minus placebo group) at week 36 . Differences may not calculate as expected because of rounding. $\mathrm{Cl}$ denotes confidence interval

*Missing data handled using control-based pattern imputation

**Refer to Fig. 3. $p$ value is obtained from a stratified Cochran-Mantel-Haenszel (CMH) test, including stratification factors of MMSE at baseline, education level, and age group 


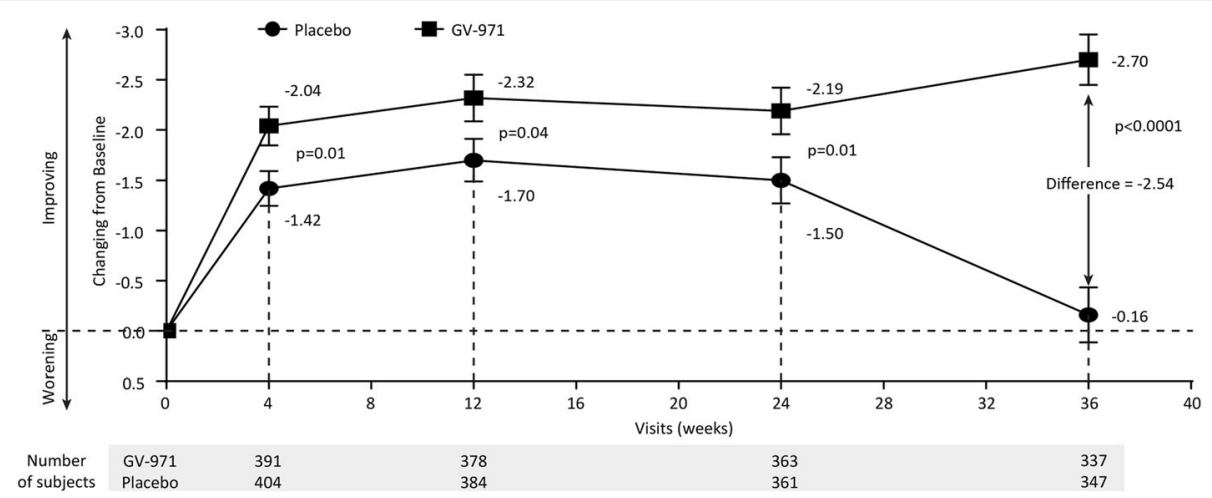

Fig. 2 Mean ADAS-Cog12 score change from baseline at weeks 4, 12, 24, and 36 (observed value). The mean change from baseline to week 36 on the ADAS-Cog12 (with scores ranging from 0 to 75 and higher scores indicating greater impairment) by full analysis set was showed. Error bars represent standard errors (SE). $p$ values are obtained from the Wilcoxon rank sum tests

between the groups (Figs. 3 and 4). The ADCS-ADL was directionally in favor of GV-971 but was not statistically different between the groups (LS difference $=0.26, p=$ 0.57 ) (Figs. 3 and 4). The NPI was directionally in favor of placebo but was not statistically different between the groups (LS difference $=0.12, p=0.80$ ) (Figs. 3 and 4).

In pre-planned exploratory analyses, we assessed the treatment effect of GV-971 in the participant groups of MMSE 11-14, 15-19, and 20-26 (Fig. 5). The adjusted difference values of the primary outcome in ADAS$\operatorname{Cog} 12$ between the groups were 4.55, 2.96, and 1.66, respectively (Fig. 5). Prespecified subgroup analyses for the change in ADAS-Cog12 score from baseline to week 36 are shown in Figure S1 (Supplementary Appendix). Significant intergroup differences in drug-placebo changes from baseline were found for all subgroups: \pm APOE 4 allele, age $(\leq 65 ;>65$ years $)$ group, sex, education level $(<6$; $>6$ years), and MMSE score ( 3 terciles). In a post hoc subgroup analyses, significant intergroup differences were detected for CIBIC+ outcome in participants with MMSE scores $11-14(p=0.017)$, effect size 1.3. (Figure $\mathrm{S} 2$ in the Appendix).

Forty-one (10.5\%) participants in the GV-971 group and $31(7.7 \%)$ participants in the placebo group were assessed by $\left[{ }^{18} \mathrm{~F}\right]$-FDG-PET. The demographic and baseline characteristics were balanced between the placebo and GV-971 sub-cohort groups. There was no intergroup difference in the changes from baseline in the predefined sROI-based global relative CMRglu on $\left[{ }^{18} \mathrm{~F}\right]-$ FDG-PET after 36 weeks of double-blind treatment.

\section{Adverse events}

In total, 307 of 407 participants (75.4\%) in the GV-971 group and 303 of $410(73.9 \%)$ in the placebo group had at least one treatment-emergent adverse event (TEAE) in the safety analysis set. Table 3 lists the TEAEs that occurred in $\geq 5 \%$ of participants. Among the common TEAEs, hyperlipidemia and nasopharyngitis were higher

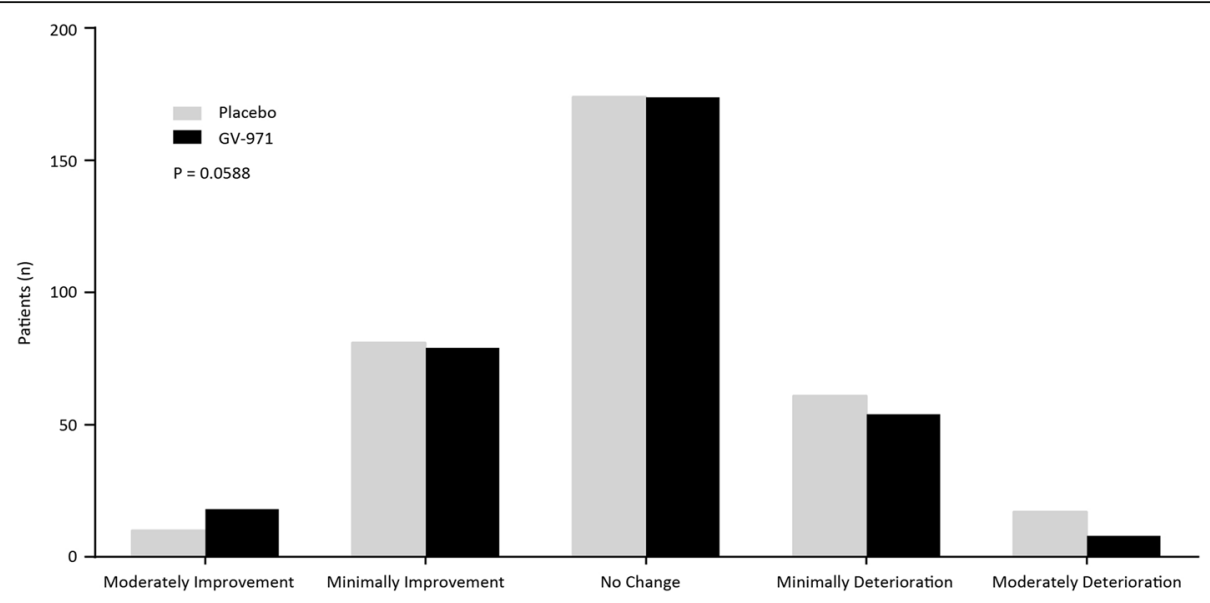

Fig. 3 Secondary outcome of CIBIC+ at week 36. $p$ value is obtained from a stratified Cochran-Mantel-Haenszel (CMH) test, including stratification factors of MMSE at baseline, education level, and age group 


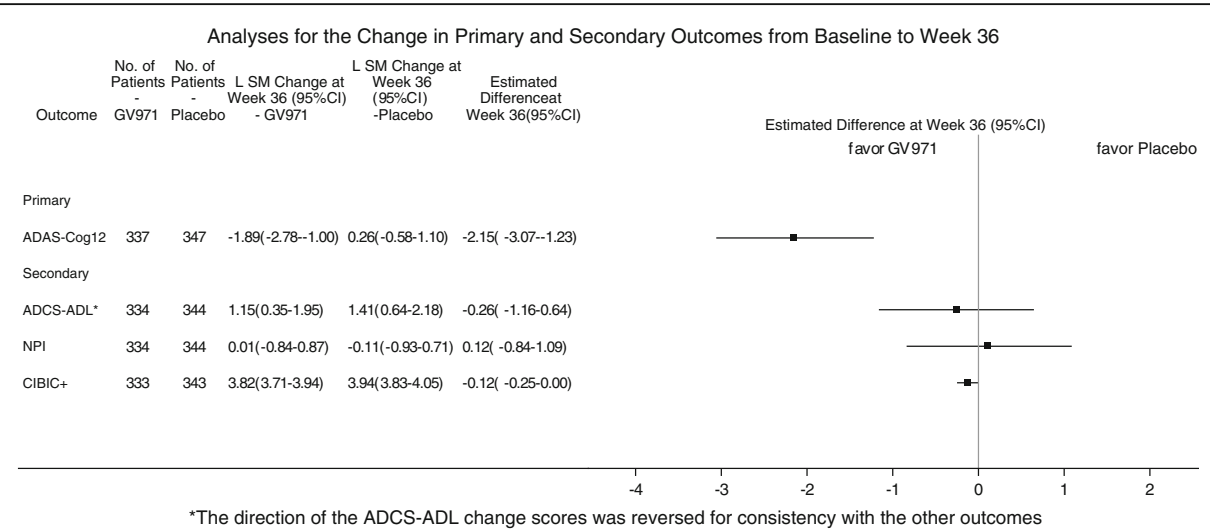

Fig. 4 Analyses for the change in primary and secondary outcomes from baseline to week $36 .{ }^{*}$ The direction of the ADCS-ADL change scores was reversed for consistency with the other outcomes

in the GV-971 group than in the placebo group. All other common TEAEs were not statistically significantly different between the two groups.

Seventy-six participants (18.7\%) in the GV-971 group and $86(20.9 \%)$ in the placebo group reported a TEAE that was related or possibly related to the trial drug according to an investigator. Fourteen participants $(3.4 \%)$ in the GV-971 group and $9(2.2 \%)$ in the placebo group had a TEAE that led to their discontinuation from the trial.

Thirty-three participants (8.1\%) in the GV-971 group and $29(7.1 \%)$ in the placebo group reported at least one serious adverse event (SAE). For the GV-971 group, the SAE of infectious pneumonia reported by one participant was determined as being possibly related to the trial drug by investigators. The remaining SAEs were determined to be not related or possibly related to the trial drug. Tables S1-1 and S1-2 in the Supplementary Appendix list all the SAEs that occurred for each treatment group.

Two participants in the GV-971 group died during the trial because of metastatic lung cancer and brain stem encephalitis (Table S2 in the Supplementary Appendix).
Examination of all listed causes of death revealed no similarities, and the deaths were not reported to be related to the drug as assessed by the site investigator.

\section{Discussion}

We report the first phase 3 clinical trial of GV-971 that demonstrated a robust, statistically significant drugplacebo difference in change from baseline favoring GV971 as measured by the ADAS-cog. The drug-placebo difference was present at the first assessment and continued throughout the 36-week trial. The separation was greatest at the endpoint of the trial between groups. In this trial, GV-971 had a therapeutic effect on patients with mild-to-moderate $\mathrm{AD}$. The effects were most pronounced in those with more severe cognitive decline. The trial demonstrated improvement above baseline suggesting that GV-971 has symptomatic effects, while the non-clinical studies support the occurrence of disease-modifying effects in concert with the symptomatic changes.

The trial was conducted according to the International Conference on Harmonization (ICH) of Drug Development Standards and Good Clinical Practice (GCP)
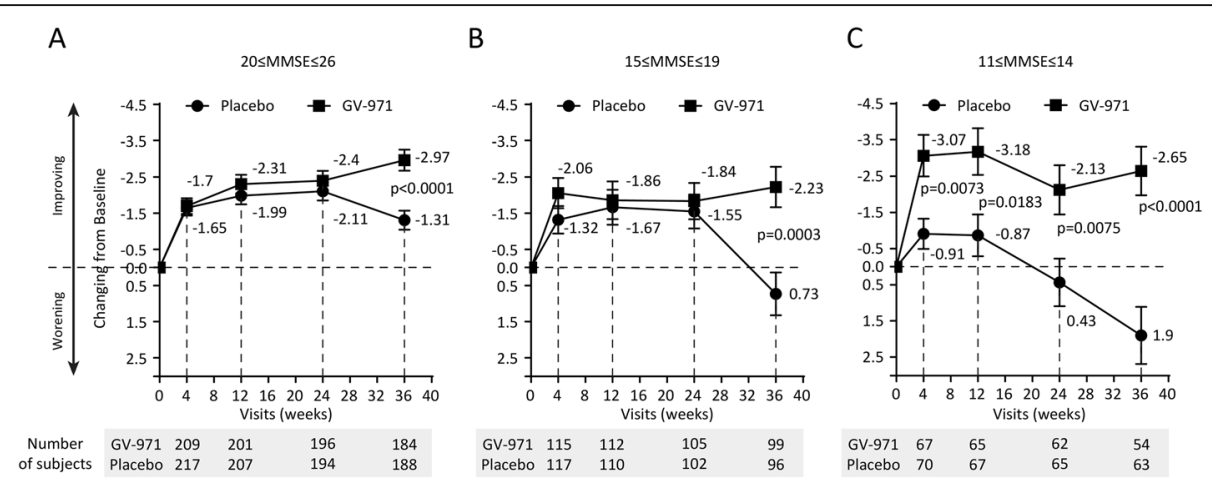

Fig. 5 Mean ADAS-Cog12 score change from baseline at weeks 4, 12, 24, and 36 (observed value) on the ADAS-Cog12 by the MMSE subgroup. Error bars represent standard errors (SE). $p$ values are obtained from the Wilcoxon rank sum tests 
Table 3 TEAEs that occurred in 5\% or more of the subjects in the study

\begin{tabular}{|c|c|c|c|c|}
\hline \multirow[t]{2}{*}{ System organ class } & \multicolumn{2}{|l|}{ Placebo, $n=410$} & \multicolumn{2}{|c|}{ GV-971 $900 \mathrm{mg}, n=407$} \\
\hline & Number of events & $n(\%)$ & Number of events & $n(\%)$ \\
\hline \multicolumn{5}{|l|}{ Infectious and infectious diseases } \\
\hline Urinary tract infection & 45 & $40(9.8 \%)$ & 31 & $30(7.4 \%)$ \\
\hline Nasopharyngitis & 26 & $23(5.6 \%)$ & 34 & $30(7.4 \%)$ \\
\hline Upper respiratory tract infection & 36 & $30(7.3 \%)$ & 29 & $23(5.7 \%)$ \\
\hline \multicolumn{5}{|l|}{ Various types of inspection } \\
\hline Elevated blood glucose & 30 & $29(7.1 \%)$ & 32 & $28(6.9 \%)$ \\
\hline \multicolumn{5}{|l|}{ Various nervous system diseases } \\
\hline Dizzy & 28 & $24(5.9 \%)$ & 26 & $23(5.7 \%)$ \\
\hline \multicolumn{5}{|l|}{ Metabolic and nutritional diseases } \\
\hline Hyperlipidemia & 15 & $14(3.4 \%)$ & 29 & $29(7.1 \%)$ \\
\hline
\end{tabular}

guidelines, was organized and monitored by an international contract research organization (CRO), required centralized reading of MRI to confirm temporal lobe atrophy consistent with $\mathrm{AD}$, and included extensive training in clinical trial methods by international vendors. GV-971 is an oligosaccharide with a novel proposed mechanism of action. In non-clinical studies, GV-971 showed a reduction of neuroinflammation in the brain by regulating the gut microbiota and reducing peripheral inflammation that may aggravate neuroinflammation. GV-971 directly binds to $A \beta$ and decreases $A \beta$ deposition in the brain [11-15]. The effect of GV-971 on the gut mirobiota and neuroinflammation is rapid, occurring within 4 weeks in animal models, which might contribute to the early therapeutic effect of GV-971 observed on the ADAS-Cog12. The effect on $A \beta$ deposition requires a longer exposure but could work synergistically with the effect on the gut mirobiota and neuroinflammation. Emerging evidence supports the concept that dysbiosis of the gut microbiota, neuroinflammation, $A \beta$ deposition, and tau hyperphosphorylation can interact to accelerate AD progression [32-35]. The therapeutic effect of GV-971 on dysbiosis of the gut microbiota, neuroinflammation, and $A \beta$ deposition may ameliorate the pathologic cascade to improve symptoms and delay disease progression with beneficial longer-term effects. The rapid decline in the placebo group at the end of the trial and the sustained response to GV-971 during the course of the trials contribute to the growing drugplacebo difference as the trial progressed. Investigation of the effects of GV-971 will require additional trials and inclusion of biomarkers to verify the mechanism of action.

In the full analysis set, none of the secondary endpoints, i.e., global function (CIBIC+), activities of daily living (ADCS-ADL) scale or behavioral symptoms (NPI), showed significant drug-placebo differences. The lack of significance on the CIBIC+, ADCS-ADL, and NPI might be attributable to the limited sample size, which was calculated based on the primary endpoint. Cultural differences may also have contributed to these negative outcomes as activities of daily living, and behavioral assessments are subject to many cultural interpretations [25]. NPI scores at baseline were very low (average score, 3 points) leaving a limited dynamic range to show measurable improvement. Finally, the relatively short duration of the trial may have contributed to the inability to show a change from baseline or drug-placebo difference.

\section{Limitations}

A limitation of our trial was the lack of requirement for the presence of a diagnostic amyloid biomarker at screening, thereby potentially allowing participants who had dementia owing to non-amyloid-related diseases to be included. Amyloid positron emission tomography was not widely available in China at the time the trial was planned and initiated. Approximately $50 \%$ of the participants in the trial were carriers of the APOE4 gene and therefore had a higher likelihood of amyloid-related disease than non-carriers $[5,36]$. Treatment benefit was evident in both APOE4 carriers and non-carriers. Structural MRI verifying the presence of temporal lobe atrophy based on central reads of the imaging supported the diagnosis of $\mathrm{AD}$. Other limitations include that the trial was conducted in one country and the external validity of the findings remains to be demonstrated. The unusually robust placebo responses observed in this trial may have been related to "trial effects," including the higher proportion of mild cases, and the excellent care delivery in trials and high participant and caregiver expectations. Similar placebo effects and benefits have been observed in other trials in China [37-40].

\section{Conclusions}

In conclusion, this phase 3 trial was well conducted by the International Conference on Harmonization of Drug 
Development and Good Clinical Practice Guidelines. The trial demonstrated a robust and sustained drugplacebo difference on the primary outcome. We plan to conduct a global phase 3 trial to assess the safety and efficacy of GV-971 in additional populations. We will collect biomarkers in these trials and will interrogate the mechanism of action of GV-971 including its effects on neuroinflammation and gut dysbiosis.

\section{Abbreviations}

AD: Alzheimer's disease; ADAS-cog12: 12-item cognitive subscale of the Alzheimer's Disease Assessment Scale; CIBIC+: Clinician's Interview-Based Impression of Change with caregiver input; ADCS-ADL: Alzheimer's Disease Cooperative Study-Activities of Daily Living; NPI: Neuropsychiatric Inventory; FDG-PET: Fluorodeoxyglucose-positron emission tomography; MRI: Magnetic resonance imaging; MMSE: Mini-Mental State Examination;

APOE: Apolipoprotein E (APOE); ALT: Alanine transaminase; AST: Aspartate transaminase; NMPA: National Medical Products Administration; GMP: Good Manufacturing Practice; IWRS: Interactive web response system; CMRglu: Cerebral metabolic rate for glucose; AEs: Adverse events; ANCOVA: Analysis of covariance; MMRM: Mixed-model repeated-measures model; FAS: Full analysis set; SUVR: Standardized uptake value ratio; APOE ع4: Apolipoprotein E epsilon 4; TEAE: Treatment-emergent adverse event; SAE: Serious adverse event; $\mathrm{ICH}$ : International Conference on Harmonization; GCP: Good Clinical Practice; CRO: Contract research organization; $A \beta$ : $\beta$ Aamyloid

\section{Supplementary Information}

The online version contains supplementary material available at https://doi. org/10.1186/s13195-021-00795-7.

Additional file 1: Supplementary figures and tables.

\section{Acknowledgements}

We thank all the investigators, participants, caregivers, and families who participated in this trial; the site investigators and personnel; the members of the steering committee; and the members of the trial team for their role in the preparation of the manuscript.

\section{Authors' contributions}

Shifu Xiao and Zhenxin Zhang were the principal investigators. Shifu Xiao, Tao Wang, Zhenxin Zhang, and Meiyu Geng designed, oversaw, and summarized this trial. Piu Chan, Tao Wang, Zhen Hong, Shuzhen Wang, Weihong Kuang, Jincai He, Xiaoping Pan, Yuying Zhou, Yong Ji, Luning Wang, Yan Cheng, Ying Peng, Qinyong Ye, Xiaoping Wang, Yuncheng Wu, Qiumin Qu, Shengdi Chen, Shuhua Li, Wei Chen, Jun Xu, Dantao Peng, Zhongxin Zhao, Yansheng Li, Junjian Zhang, Yifeng Du, Weixian Chen, Dongsheng Fan, Yong Yan, Xiaowei Liu, Wei Zhang, Benyan Luo, Wenyuan Wu, Lu Shen, Chunfeng Liu, Peixian Mao, Qiumei Wang, Qianhua Zhao, Qihao Guo, Yongtao Zhou, Yi Li, Lijun Jiang, Wenwei Ren, Yingjun Ouyang, Yan Wang, Shuai Liu, Jianjun Jia, Nan Zhang, Zhonglin Liu, Raoli He, Tingyi Feng, Wenhui Lu, Huidong Tang, Ping Gao, Yingchun Zhang, Lanlan Chen, Lei Wang, You Yin, Qun Xu, Jinsong Xiao, Lin Cong, Xi Cheng, Hui Zhang, Dan Gao, Minghua Xia, Tenghong Lian, Guoping Peng, Xu Zhang, Bin Jiao, $\mathrm{Hua} \mathrm{Hu}$, and Xueyan Chen were the trial investigators. Yihui Guan, Ruixue Cui, Qiu Huang, and Kewei Chen contributed to the imaging data collection and analysis. Xianliang Xin, Hongjian Chen, Yu Ding, Jing Zhang, and Jian Ding contributed to the research implementation. Shifu Xiao, Meiyu Geng, Tao Wang, Teng Feng, Marc Cantillon, Kewei Chen, and Jeffrey L. Cummings contributed to the analysis and manuscript writing. All authors reviewed the manuscript. The authors read and approved the final manuscript.

\section{Funding}

This trial was funded by the Shanghai Green Valley Pharmaceuticals (SHGV) and National Science and Technology Major Project for Investigational New Drugs (2011ZX09101-003-01, 2015ZX09101003, 2018ZX09711002-015).

\section{Availability of data and materials}

The datasets used and/or analyzed during the present study are available from the corresponding author on reasonable request.

\section{Declarations}

\section{Ethics approval and consent to participate}

The trial protocol was approved by the Ethics Review Board of Shanghai Mental Health Center (Shanghai, China). The trial protocol was approved by the Institutional Review Boards of all participating sites. The Clinical New Drug Research and Development Team of the Department of Psychogeriatrics of Shanghai Mental Health Center and the sponsor designed the study in consultation with academic advisors. All participants or their representatives provided written informed consent before participation in the trial.

\section{Consent for publication}

All authors approved the final version of the manuscript for submission.

\section{Competing interests}

Shifu Xiao and Zhenxin Zhang have been the provisional consultants of Pfizer, Lilly, Novartis, Roche, GSK, Johnson \& Johnson, Lundbeck, Biogen, and Green Valley and received honorariums. Xianliang Xin, Hongjian Chen, Yu Ding, Jing Zhang, and Marc Cantillon were full-time employees of Shanghai Green Valley Pharmaceutical Co., Ltd. Kewei Chen was a full-time employee of Shanghai Green Valley Pharmaceutical Co., Ltd. at the time the $\left[{ }^{18} \mathrm{~F}\right]$-FDGPET data was analyzed. Currently, he is a full-time employee of Banner Health to which Banner Alzheimer's Institute belongs, and serves as a paid consultant to Shanghai Green Valley Pharmaceutical Co., Ltd. Jeffrey L. Cummings has provided consultation to Acadia, Actinogen, Alkahest, Alzheon, Avanir, Axsome, BiOasis Technologies, Biogen, Cerecin, CNS Innovations, Cortexyme, EIP, Eisai, Genentech, Green Valley, Grifols, Hisun, Idorsia, Merck, Otsuka, Resverlogix, Roche, Samus, Samumed, Sunovion, Suven, and United Neuroscience pharmaceutical and assessment companies.

\section{Author details}

${ }^{1}$ Department of Geriatric Psychiatry, Shanghai Mental Health Center, Shanghai Jiaotong University School of Medicine, Shanghai, China. ${ }^{2}$ Alzheimer's Disease and Related Disorders Center, Shanghai Jiaotong University, 600 South Wan Ping Road, Shanghai 200030, China. ${ }^{3}$ Xuanwu Hospital Capital Medical University, Beijing, China. ${ }^{4}$ Huashan Hospital, Fudan University, Shanghai, China. ${ }^{5}$ Qilu Hospital of Shandong University, Ji'nan, China. ${ }^{6}$ West China Hospital of Sichuan University, Chengdu, China. ${ }^{7}$ The First Affiliated Hospital of Wenzhou Medical University, Wenzhou, China. ${ }^{8}$ Guangzhou First People's Hospital, School of Medicine, South China University of Technology, Guangzhou, China. 'Tianjin Huanhu Hospital, Huanhu Hospital Affiliated to Nankai University, Tianjin, China. ${ }^{10}$ Department of Geriatric Neurology of PLA General Hospital, Beijing, China. ${ }^{11}$ Department of Neurology, Tianjin Medical University General Hospital, Tianjin, China.

${ }^{12}$ Sun Yat-Sen Memorial Hospital, Sun Yat-Sen University, Guangzhou, China. ${ }^{13}$ Fujian Medical University Union Hospital, Fuzhou, China. ${ }^{14}$ Department of Neurology, Shanghai General Hospital, Shanghai Jiao Tong University School of Medicine, Shanghai, China. ${ }^{15}$ Department of Neurology, The First Affiliated Hospital of Xi'an Jiaotong University, Xi'an, China. ${ }^{16}$ Department of Neurology, Ruijin Hospital, Shanghai Jiaotong University School of Medicine, Shanghai, China. ${ }^{17}$ Beijing Hospital, Beijing, China. ${ }^{18}$ Department of Psychiatry, Sir Run Run Shaw Hospital, Zhejiang University School of Medicine and Key Laboratory of Medical Neurobiology of Zhejiang Province, Hangzhou, China. ${ }^{19}$ Northern Jiangsu People's Hospital, Yangzhou, China. ${ }^{20}$ Department of Neurology, China-Japan Friendship Hospital, Beijing, China. ${ }^{21}$ Shanghai Changzheng Hospital, Shanghai, China. ${ }^{22}$ Renji Hospital, Shanghai Jiaotong University School of Medicine, Shanghai, China. ${ }^{23}$ Department of Neurology, Zhongnan Hospital of Wuhan University, Wuhan, China. ${ }^{24}$ Shandong Provinical Hospital affiliated to Shandong University, Ji'nan, China. ${ }^{25}$ Jiangsu Province People's Hospital, Nanjing, China. ${ }^{26}$ Peking University Third Hospital, Beijing, China. ${ }^{27}$ The First Affiliated Hospital of Chongqing Medical University, Chongqing, China. ${ }^{28}$ Department of Geriatric psychiatry, Wuxi Mental Health Center, Wuxi, China. ${ }^{29}$ Department of Neurology, Beijing Tiantan Hospital, Capital Medical University, Beijing, China. ${ }^{30}$ The First Affiliated Hospital, Zhejiang University School of Medicine, Hangzhou, China. ${ }^{31}$ Tongji Hospital of Tongji University, Shanghai, China. 
${ }^{32}$ Xiangya Hospital Central South University, Changsha, China. ${ }^{33}$ The Second Affiliated Hospital of Soochow University, Suzhou, China. ${ }^{34}$ Beijing An Ding Hospital, Capital Medical University, Beijing, China. ${ }^{35}$ Peking Union Medical College Hospital, No. 1 Shuaifuyuan, Beijing 100730, China. ${ }^{36}$ Med-X Research Institution, Shanghai Jiao Tong University, Shanghai, China. ${ }^{37}$ Shanghai Green Valley Pharmaceutical Co. Ltd., No. 421, Niudun Road, Shanghai, China. ${ }^{38}$ Banner Alzheimer's Institute, Phoenix, AZ, USA. ${ }^{39}$ Chamberrs-Grundy Center for Transformative Neuroscience, Department of Brain Health, School of Integrated Health Sciences, University of Nevada, Las Vegas, USA. ${ }^{40}$ State Key Laboratory of Drug Research, Shanghai Institute of Materia Medica, Chinese Academy of Sciences, 555 Zu chong zhi Road, Nevada, China.

Received: 12 November 2020 Accepted: 22 February 2021 Published online: 17 March 2021

\section{References}

1. Zhang Y, Xu Y, Nie H, Lei T, Wu Y, Zhang L, Zhang M. Prevalence of dementia and major dementia subtypes in the Chinese populations: a meta-analysis of dementia prevalence surveys, 1980-2010. J Clin Neurosci. 2012;19(10):1333-7.

2. Chan KY, Wang W, Wu JJ, Liu L, Theodoratou E, Car J, Middleton L, Russ TC, Deary IJ, Campbell H, et al. Epidemiology of Alzheimer's disease and other forms of dementia in China, 1990-2010: a systematic review and analysis. Lancet. 2013;381(9882):2016-23.

3. Rogers SL, Farlow MR, Doody RS, Mohs R, Friedhoff LT. A 24-week, doubleblind, placebo-controlled trial of donepezil in patients with Alzheimer's disease. Donepezil Study Group. Neurology. 1998;50(1):136-45.

4. Rosler M, Anand R, Cicin-Sain A, Gauthier S, Agid Y, Dal-Bianco P, Stahelin $H B$, Hartman R, Gharabawi M. Efficacy and safety of rivastigmine in patients with Alzheimer's disease: international randomised controlled trial. BMJ. 1999;318(7184):633-8.

5. Wilcock GK, Lilienfeld S, Gaens E. Efficacy and safety of galantamine in patients with mild to moderate Alzheimer's disease: multicentre randomised controlled trial. Galantamine International-1 Study Group. BMJ. 2000; 321(7274):1445-9.

6. van Dyck CH, Tariot PN, Meyers B, Malca Resnick E, Memantine MEMMDSG. A 24-week randomized, controlled trial of memantine in patients with moderateto-severe Alzheimer disease. Alzheimer Dis Assoc Disord. 2007;21(2):136-43.

7. Doody RS, Thomas RG, Farlow M, Iwatsubo T, Vellas B, Joffe S, Kieburtz K, Raman R, Sun X, Aisen PS, et al. Phase 3 trials of solanezumab for mild-tomoderate Alzheimer's disease. N Engl J Med. 2014;370(4):311-21.

8. Salloway S, Sperling R, Fox NC, Blennow K, Klunk W, Raskind M, Sabbagh M, Honig LS, Porsteinsson AP, Ferris S, et al. Two phase 3 trials of bapineuzumab in mild-to-moderate Alzheimer's disease. N Engl J Med. 2014;370(4):322-33.

9. Cummings J, Lee G, Ritter A, Zhong K. Alzheimer's disease drug development pipeline: 2018. Alzheimers Dement (N Y). 2018:4:195-214.

10. Wang T. New drug research and development for Alzheimer's pathology: present and prospect. Shanghai Arch Psychiatry. 2017;29(4):237-9.

11. Wang X, Sun G, Feng T, Zhang J, Huang X, Wang T, Xie Z, Chu X, Yang J, Wang $H$, et al. Sodium oligomannate therapeutically remodels gut microbiota and suppresses gut bacterial amino acids-shaped neuroinflammation to inhibit Alzheimer's disease progression. Cell Res. 2019;29(10):787-803.

12. Kong LN, Geng MY, Mu L, Xin XL, Yang N, Zuo PP. Effects of acidic oligose on differentially expressed genes in the mice model of Alzheimer's disease by microarray. Yao xue xue bao. 2005;40(12):1105-9.

13. Fan Y, Hu J, Li J, Yang Z, Xin X, Wang J, Ding J, Geng M. Effect of acidic oligosaccharide sugar chain on scopolamine-induced memory impairment in rats and its related mechanisms. Neurosci Lett. 2005;374(3):222-6.

14. Hu J, Geng M, Li J, Xin X, Wang J, Tang M, Zhang J, Zhang X, Ding J. Acidic oligosaccharide sugar chain, a marine-derived acidic oligosaccharide, inhibits the cytotoxicity and aggregation of amyloid beta protein. J Pharmacol Sci. 2004;95(2):248-55.

15. Jiang RW, Du XG, Zhang X, Wang X, Hu DY, Meng T, Chen YL, Geng MY, Shen JK. Synthesis and bioassay of beta-(1,4)-D-mannans as potential agents against Alzheimer's disease. Acta Pharmacol Sin. 2013;34(12):1585-91.

16. Wang $T$, Kuang W, Chen W, Xu W, Zhang L, Li Y, Li H, Peng Y, Chen Y, Wang $B$, et al. A phase II randomized trial of sodium oligomannate in Alzheimer's dementia. Alzheimers Res Ther. 2020;12(1):110.

17. Folstein MF, Folstein SE, McHugh PR. "Mini-mental state". A practical method for grading the cognitive state of patients for the clinician. J Psychiatr Res. 1975;12(3):189-98.
18. McKhann G, Drachman D, Folstein M, Katzman R, Price D, Stadlan EM. Clinical diagnosis of Alzheimer's disease: report of the NINCDS-ADRDA Work Group under the auspices of Department of Health and Human Services Task Force on Alzheimer's Disease. Neurology. 1984;34(7):939-44.

19. Katzman R, Zhang MY, Ouang Ya Q, Wang ZY, Liu WT, Yu E, Wong SC, Salmon DP, Grant I. A Chinese version of the Mini-Mental State Examination; impact of illiteracy in a Shanghai dementia survey. J Clin Epidemiol. 1988;41(10):971-8.

20. Hachinski VC, lliff LD, Zilhka E, Du Boulay GH, McAllister VL, Marshall J, Russell RW, Symon L. Cerebral blood flow in dementia. Arch Neurol. 1975;32(9):632-7.

21. Hamilton M. A rating scale for depression. J Neurol Neurosurg Psychiatry. 1960;23:56-62.

22. Cho H, Kwon JH, Seo HJ. Medial temporal lobe atrophy in vascular dementia: visual temporal lobe rating scale. Arch Gerontol Geriatr. 2009;48(3):415-8.

23. Inzitari D, Simoni M, Pracucci G, Poggesi A, Basile AM, Chabriat H, Erkinjuntti $T$, Fazekas F, Ferro JM, Hennerici $M$, et al. Risk of rapid global functional decline in elderly patients with severe cerebral age-related white matter changes: the LADIS study. Arch Intern Med. 2007;167(1):81-8.

24. Boothby $\mathrm{H}$, Mann AH, Barker A. Factors determining interrater agreement with rating global change in dementia: the cibic-plus. Int J Geriatr Psychiatry. 1995;10(12):1037-45.

25. Reisberg B. FS: Clinician's interview-based impression of change-plus; in Kelly C, Newton-Howes G (eds): guide to assessment scales in dementia. London: Science Press; 1994.

26. Galasko D, Bennett D, Sano M, Ernesto C, Thomas R, Grundman M, Ferris S. An inventory to assess activities of daily living for clinical trials in Alzheimer's disease. The Alzheimer's Disease Cooperative Study. Alzheimer Dis Assoc Disord. 1997;11(Suppl 2):S33-9.

27. Cummings JL, Mega M, Gray K, Rosenberg-Thompson S, Carusi DA, Gornbein J. The Neuropsychiatric Inventory: comprehensive assessment of psychopathology in dementia. Neurology. 1994;44(12):2308-14.

28. Wang T, Xiao S, Li X, Wang H, Liu Y, Su N, Fang Y. Reliability and validity of the Chinese version of the neuropsychiatric inventory in mainland China. Int J Geriatr Psychiatry. 2012;27(5):539-44.

29. Rosen WG, Mohs RC, Davis KL. A new rating scale for Alzheimer's disease. Am J Psychiatry. 1984;141(11):1356-64.

30. Chen K, Langbaum JB, Fleisher AS, Ayutyanont N, Reschke C, Lee W, Liu X, Bandy D, Alexander GE, Thompson PM, et al. Twelve-month metabolic declines in probable Alzheimer's disease and amnestic mild cognitive impairment assessed using an empirically pre-defined statistical region-ofinterest: findings from the Alzheimer's Disease Neuroimaging Initiative. Neurolmage. 2010;51(2):654-64.

31. van Dyck CH, Nygaard HB, Chen K, Donohue MC, Raman R, Rissman RA, Brewer JB, Koeppe RA, Chow TW, Rafii MS, et al. Effect of AZD0530 on Cerebral Metabolic Decline in Alzheimer Disease: A Randomized Clinical Trial. JAMA Neurol. 2019;76(10):1219-29.

32. Seo DO, Holtzman DM. Gut Microbiota: From the Forgotten Organ to a Potential Key Player in the Pathology of Alzheimer's Disease. J Gerontol A Biol Sci Med Sci. 2020;75(7):1232-41.

33. Kim MS, Kim Y, Choi H, Kim W, Park S, Lee D, Kim DK, Kim HJ, Choi H, Hyun DW, et al. Transfer of a healthy microbiota reduces amyloid and tau pathology in an Alzheimer's disease animal model. Gut. 2020;69(2):283-94.

34. Chen C, Ahn EH, Kang SS, Liu X, Alam A, Ye K. Gut dysbiosis contributes to amyloid pathology, associated with C/EBPbeta/AEP signaling activation in Alzheimer's disease mouse model. Sci Adv. 2020;6(31):eaba0466.

35. Cryan JF, O'Riordan KJ, Sandhu K, Peterson V, Dinan TG. The gut microbiome in neurological disorders. Lancet Neurol. 2020;19(2):179-94.

36. Cummings JL, Morstorf T, Zhong K. Alzheimer's disease drug-development pipeline: few candidates, frequent failures. Alzheimers Res Ther. 2014;6(4):37.

37. Chiu HF, Lam LC. Relevance of outcome measures in different cultural groups--does one size fit all? Int Psychogeriatr. 2007;19(3):457-66.

38. Geuter S, Koban L, Wager TD. The cognitive neuroscience of placebo effects: concepts, predictions, and physiology. Annu Rev Neurosci. 2017;40:167-88.

39. Friesen $P$, Blease $C$. Placebo effects and racial and ethnic health disparities: an unjust and underexplored connection. J Med Ethics. 2018;44(11):774-81.

40. Tachibana $Y$, Narukawa M. Investigation of influencing factors on higher placebo response in East Asian versus Western clinical trials for partial epilepsy: a meta-analysis. Clin Drug Invest. 2013;33(5):315-24.

\section{Publisher's Note}

Springer Nature remains neutral with regard to jurisdictional claims in published maps and institutional affiliations. 